\title{
éditionsOCDE
}

\section{LES RÉSEAUX DE LA COOPÉRATION TRANSFRONTALIÈRE EN AFRIQUE DE L'OUEST}

\section{NOTES OUEST-AFRICAINES}

Juin $2017 \mathbf{N}^{\circ} \mathbf{0 6}$

Secrétariat du

Club 



\section{LES RÉSEAUX DE LA COOPÉRATION TRANSFRONTALIĖRE EN AFRIQUE DE L'OUEST}

Cette note a été rédigée par

OLIVIER WALTHER

Université du Sud-Danemark 


\section{NOTES OUEST-AFRICAINES}

La série Notes ouest-africaines analyse les dynamiques socio-économiques, politiques et sécuritaires que traverse l'Afrique dans une perspective régionale et multidisciplinaire. Elle cherche à stimuler la discussion, rassembler les informations et mieux anticiper les transformations en cours pour les politiques à venir. La série vise à partager des études avec une large audience d'experts, de praticiens du développement, de décisionnaires et de lecteurs avertis. Les Notes sont disponibles en anglais et/ou en français ; les résumés dans les deux langues. Initiées par le Club du Sahel et de l'Afrique de l'Ouest (CSAO) pour éclairer les enjeux ouest-africains, ces analyses sont préparées par son Secrétariat, ses membres et partenaires, les autres départements de l'OCDE, des organisations internationales et autres experts et chercheurs.

En savoir plus sur le Club du Sahel et de l'Afrique de l'Ouest : http://www.oecd.org/fr/csao.

Merci de citer cet ouvrage comme suit:

Walther, O. (2017), (c Les réseaux de la coopération transfrontalière en Afrique de l'Ouest ), Notes ouest-africaines, $\mathrm{N}^{\circ} 06$, Éditions OCDE, Paris.

http://dx.doi.org/10.1787/b7ad4957-fr

Contact auteur : ow@sam.sdu.dk

ISSN 2414-2026

Les documents de travail de l'OCDE ne doivent pas être présentés comme exprimant les vues officielles de l'OCDE ou de ses pays membres. Les opinions exprimées et les arguments employés sont ceux des auteurs.

Ce document et toute carte qu'il peut comprendre ne préjugent en rien du statut de tout territoire, de la souveraineté s'exerçant sur ce dernier, du tracé des frontières et limites internationales, et du nom de tout territoire, ville ou région.

Les documents de travail exposent des résultats préliminaires ou des travaux de recherche en cours menés par l'auteur/les auteurs et sont publiés pour stimuler le débat sur un large éventail de questions sur lesquelles l'OCDE travaille. Les commentaires sur les documents de travail sont bienvenus et peuvent être adressés au Club du Sahel et de l'Afrique de l'Ouest, OCDE, 2 rue André-Pascal, 75775 Paris Cedex 16, France.

Autorisé pour publication par Laurent Bossard, Directeur, Secrétariat du Club du Sahel et de l'Afrique de l'Ouest (CSAO/OCDE).

(C) OCDE 2017

Vous êtes autorisés à copier, télécharger ou imprimer du contenu OCDE pour votre utilisation personnelle. Vous pouvez inclure des extraits des publications, des bases de données et produits multimédia de l'OCDE dans vos documents, présentations, blogs, sites Internet et matériel d'enseignement, sous réserve de faire mention de la source OCDE et du copyright. Les demandes pour usage commercial ou de traduction devront être adressées à rights@oecd.org. 


\title{
RÉSUMÉ
}

Longtemps considérées comme des obstacles artificiels hérités de la décolonisation, les frontières ouest-africaines sont aujourd'hui au cœur des politiques visant à favoriser les échanges inter-régionaux et à lutter contre l'instabilité politique. Cette redécouverte des marges de l'État a favorisé la multiplication d'initiatives institutionnelles destinées à favoriser la coopération entre États, régions et communes tout en assurant la protection et la promotion des intérêts et droits des populations frontalières. En dépit de ces initiatives régionales, le fonctionnement effectif de la coopération transfrontalière demeure encore largement inconnu en Afrique de l'Ouest. L'objectif de cette note est de combler cette lacune en analysant la structure sociale et la géographie des réseaux de gouvernance ouest-africains. Sur la base de cette analyse structurelle et géographique sont formulées des recommandations politiques visant à mettre en œuvre des politiques plus territorialisées, plus attentives aux relations entre acteurs impliqués dans la coopération, et plus spécifiquement ajustées aux contraintes et opportunités ouest-africaines.

Mots clés : coopération transfrontalière, réseaux, gouvernance, intégration régionale, Afrique de l'Ouest Code JEL : R58, O43, O18, O550, O190

\begin{abstract}
À PROPOS DE L'AUTEUR
Olivier J. Walther est professeur associé en sciences politiques à l’Université du Sud-Danemark et professeur invité à la Division des affaires globales de l’Université d’État du New Jersey - Rutgers.

Titulaire d'un doctorat en géographie de l'Université de Lausanne, Dr Walther a orienté ses recherches sur le commerce, la coopération transfrontalière et le terrorisme en Afrique de l'Ouest en utilisant l'analyse des réseaux sociaux. Ses travaux ont été financés par la Commission européenne, le Programme alimentaire mondial, l'OCDE, l'Observatoire en réseau de l'aménagement du territoire européen, les gouvernements du Luxembourg et du Danemark, et la Fondation Carlsberg. Il est rédacteur pour l'Afrique du Journal of Borderlands Studies et membre du comité exécutif de l'African Borderlands Research Network (ABORNE).
\end{abstract}





\section{TABLE DES MATIÈRES}

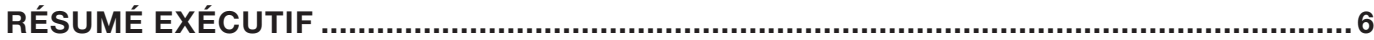

ESPACES FRONTALIERS ET RÉSEAUX DE GOUVERNANCE.......................................... 7

TROIS DIMENSIONS DE LA COOPÉRATION TRANSFRONTALIĖRE.................................. 8

Le potentiel : où la coopération transfrontalière pourrait-elle se développer ?..................8

La structure actuelle : où la coopération transfrontalière se développe-t-elle ?...............11

La vision : où la coopération transfrontalière devrait-elle se développer ?.......................16

CONJUGUER LES TROIS DIMENSIONS DE LA COOPÉRATION TRANSFRONTALIÈRE... 21

Un potentiel de coopération inexploité ...........................................................................21

Des réseaux de gouvernance peu développés ..............................................................22

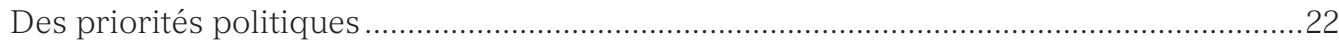

LE FUTUR DE LA COOPÉRATION TRANSFRONTALIĖRE..............................................22

Adapter les politiques aux spécificités des régions ..................................................22

Quel modèle d'intégration entre institutions et interactions fonctionnelles ? ..................23

APPRÉHENDER LA COOPÉRATION TRANSFRONTALIÈRE DE MANIÈRE

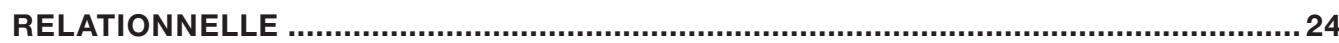

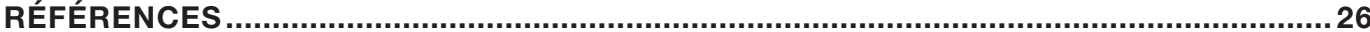




\section{RÉSUMÉ EXÉCUTIF}

es frontières ouest-africaines sont aujourd'hui au cœur des politiques visant à favoriser L les échanges régionaux et à lutter contre l'instabilité politique. Cette redécouverte des marges de l'État a favorisé la multiplication d'initiatives institutionnelles destinées à favoriser la coopération entre États, régions et communes. En dépit de ces initiatives, le fonctionnement effectif de la coopération transfrontalière demeure encore largement inconnu en Afrique de l'Ouest. Si de nombreuses études décrivent en effet les principes législatifs et institutionnels de la coopération, la structure des réseaux de gouvernance qui relient les organisations et les individus impliqués dans les projets transfrontaliers n’ont jusqu'ici fait l'objet d'aucune analyse systématique. Les spécificités géographiques des espaces frontaliers et la manière dont les frontières affectent le fonctionnement de ces réseaux de gouvernance sont également peu documentées, malgré leur importance cruciale dans la mise en œuvre des projets transfrontaliers.

L'objectif de cette note issue des travaux du Secrétariat du Club du Sahel et de l'Afrique de l'Ouest/OCDE sur la coopération transfrontalière et les réseaux de gouvernance en Afrique de l'Ouest est de combler cette lacune en analysant à la fois la structure sociale et la géographie des réseaux de gouvernance ouest-africains. Sur la base d'une analyse des réseaux sociaux conduite auprès de 137 acteurs impliqués dans la coopération locale et régionale sont formulées trois recommandations politiques.

L'analyse suggère tout d'abord que les politiques transfrontalières devraient s'appuyer sur la grande diversité des régions d’Afrique de l’Ouest. Ces politiques territorialisées doivent tenir compte de la variété des besoins, des systèmes institutionnels et des niveaux de développement des régions ouest-africaines et fournir des biens publics adaptés aux problèmes socio-économiques spécifiques de chaque région. La forte hétérogénéité des espaces frontaliers ouest-africains suggère que les investissements soient concentrés dans les régions qui possèdent le plus haut potentiel, ont déjà mis en œuvre des réseaux de gouvernance ou sont reconnues comme prioritaires par les décideurs politiques.

Les travaux montrent ensuite que la vision politique de la coopération transfrontalière véhiculée en Afrique de l'Ouest emprunte à deux grands modèles d'intégration : le modèle d'inspiration européenne dans lequel la priorité est donnée aux structures institutionnelles et le modèle d'inspiration anglo-saxonne axé sur les interactions entre acteurs socioéconomiques. Dans un contexte marqué à la fois par une certaine prolifération d’organisations intergouvernementales et par une prépondérance des interactions informelles, il n'est pas certain qu'aucun de ces modèles ne soit réellement adapté aux opportunités et contraintes auxquelles font face les acteurs de la coopération en Afrique de l'Ouest. À long terme, le succès de la coopération transfrontalière repose sur la mise en place d'un modèle d'intégration qui intègre plus étroitement les spécificités socio-économiques et politiques de la région.

Finalement, les travaux montrent que la coopération transfrontalière devrait être plus systématiquement appréhendée de manière relationnelle, c'est-à-dire en considérant les interactions entretenues entre ses acteurs (réseaux d'information et de pouvoir). Une attention exclusivement portée aux attributs institutionnels des organisations régionales, des États et des collectivités locales masque le fonctionnement réel de la coopération transfrontalière, dont l'efficacité repose bien souvent sur des relations interpersonnelles entre acteurs de nature très différente. L'analyse des réseaux sociaux constitue à ce titre un outil de recherche adapté à la compréhension d'organisations sociales fluides (fondées sur des rapports de flux), comme les décideurs impliqués dans la coopération transfrontalière. La possibilité de visualiser les relations effectivement entretenues au sein d'un groupe social , les brokers, ou les effets frontières, offre en outre un levier d'autonomisation pour les communautés locales et les organisations non gouvernementales ainsi qu'une aide à l'opérationnalisation pour les organisations internationales et les gouvernements. 
Encore peu appliqué au champ du développement, ce genre d'approche pourrait trouver des applications nouvelles à d'autres champs relationnels par nature, comme le commerce, les migrations, ou les conflits, pour autant que des données relationnelles sur les acteurs sociaux soient plus systématiquement collectées.

\section{ESPACES FRONTALIERS ET RÉSEAUX DE GOUVERNANCE}

- es quelque 32000 kilomètres de frontières terrestres qui découpent l'Afrique de — l'Ouest de Dakar à N’Djamena n'ont jamais, depuis les années 60, été aussi centrales à la mise en œuvre de politiques de développement. Longtemps considérées comme des obstacles artificiels hérités de la décolonisation, ces frontières sont aujourd'hui au cœur des politiques visant à favoriser les échanges régionaux et à lutter contre l'instabilité politique. Les dynamiques transfrontalières constituent en effet une dimension incontournable des flux commerciaux - qui subvertissent les législations nationales -, comme des trafics illicites et du terrorisme - qui jouent de la grande porosité des frontières pour faire circuler armes, drogues et migrants ou commettre des attaques dans les pays voisins.

Cette redécouverte des marges de l'État favorise la multiplication d'initiatives institutionnelles destinées à promouvoir la coopération entre États, régions et communes tout en assurant la protection et la promotion des intérêts et droits des populations frontalières. Depuis le discours pionnier du président malien Alpha Oumar Konaré sur les ( pays-frontières ») en 2002, la Communauté économique des États de l'Afrique de l'Ouest (CEDEAO) et l'Union africaine (UA) ont initié un programme spécifiquement dédié aux frontières. En 2011, l’Union économique et monétaire ouest-africaine (UEMOA) a inauguré une opération pilote avec le premier des Postes de contrôle juxtaposés aux frontières prévus entre les États membres, à Sinkansé (frontière Burkina Faso - Togo). En 2014, l'adoption de la Convention de Niamey sur la coopération transfrontalière constitue une avancée importante en matière de prise de conscience du besoin d'une législation adaptée pour le développement des activités transfrontalières.

En dépit de ces initiatives régionales, le fonctionnement effectif de la coopération transfrontalière demeure encore largement inconnu en Afrique de l'Ouest. Si de nombreuses études décrivent en effet les principes législatifs et institutionnels de la coopération (CSAO/ OCDE, 2007 ; ARFE, 2012 ; Enda Diapol, 2007), la structure des réseaux de gouvernance qui relie les organisations et les individus impliqués dans les projets transfrontaliers n’a jusqu'ici fait l'objet d'aucune analyse systématique. Les spécificités géographiques des espaces frontaliers et la manière dont les frontières affectent le fonctionnement de ces réseaux de gouvernance sont également peu documentées, malgré leur importance cruciale dans la mise en œuvre des projets transfrontaliers.

L'objectif de cette note issue des travaux du CSAO sur la coopération transfrontalière et les réseaux de gouvernance en Afrique de l'Ouest (OCDE/CSAO, 2017) est de combler cette lacune en analysant à la fois la structure sociale et la géographie des réseaux de gouvernance ouest-africains. Sur la base de cette analyse structurelle et géographique sont formulées des recommandations politiques visant à mettre en œuvre des politiques plus territorialisées, plus attentives aux relations entre acteurs impliqués dans la coopération, et plus spécifiquement adaptées aux contraintes et opportunités ouest-africaines. 


\section{TROIS DIMENSIONS DE LA COOPÉRATION TRANSFRONTALIÈRE}

a coopération transfrontalière est un processus complexe d'intégration pouvant être décomposé en trois dimensions, selon que l'analyse porte sur le potentiel des régions frontalières en matière de coopération, la structure actuelle des réseaux de gouvernance transfrontaliers ou la vision qu'ont les décideurs politiques de la coopération transfrontalière.

\section{Le potentiel : où la coopération transfrontalière pourrait-elle se développer ?}

Le potentiel des régions ouest-africaine à collaborer au travers des frontières nationales constitue la première dimension de la coopération. En s'appuyant sur une analyse cartographique de sept indicateurs politiques et socio-économiques (Tableau 1), il est possible d'examiner dans quelle mesure l'existence de disparités sociales, économiques et politiques constitue une source de synergies pour la coopération transfrontalière ou, au contraire, une barrière aux initiatives transfrontalières. Certains indicateurs, comme le potentiel de population (Carte 2), se rapportent aux interactions qui relient les régions frontalières les unes aux autres : on mesure par exemple le nombre de personnes qui peuvent être théoriquement accessibles depuis les marchés frontaliers en moins de quatre heures compte tenu du réseau routier actuel du continent. D'autres indicateurs, comme les différentiels de pauvreté entre régions contiguës, se rapportent au processus de convergence entre régions frontalières. On mesure alors la tendance des régions à l’homogénéisation de leurs attributs.

Tableau 1

Indicateurs d'intégration régionale

\begin{tabular}{lll}
\hline \multicolumn{1}{c}{ Indicateur } & \multicolumn{1}{c}{ Définition } & Type d'intégration \\
\hline Population & $\begin{array}{l}\text { Nombre de personnes pouvant potentiellement être atteintes } \\
\text { depuis les marchés frontaliers en moins de quatre heures }\end{array}$ & Interaction \\
\hline Ressources hydrauliques & Existence d'eaux de surface et d'aquifères partagés & Interaction \\
\hline Ressources agricoles et pastorales & $\begin{array}{l}\text { Existence de bassins de production agricoles et de circuits de } \\
\text { transhumance partagés }\end{array}$ & Interaction \\
\hline Langues & Existence de discontinuités majeures entre langues & Convergence \\
\hline Statut légal des frontières & Existence de frontières clairement délimitées et démarquées & Convergence \\
\hline Stabilité politique & Existence de disputes frontalières, de conflits et de groupes & \\
& extrémistes violents transnationaux & Interaction \\
\hline Pauvreté & Différence de taux de pauvreté entre régions contiguës & Convergence \\
\hline
\end{tabular}

Source : OCDE/CSA0 2017

Létude combinée des indicateurs d'intégration régionale conduit à une synthèse du potentiel de coopération des frontières ouest-africaines. Un nombre de points est attribué à chaque segment de frontière séparant les 179 régions administratives de l'Afrique de l'Ouest en fonction de l'intensité - haute, moyenne, ou faible - du potentiel de coopération transfrontalière observé (Tableau 2). Par exemple, le potentiel de coopération est considéré comme maximum quand une région présente des frontières clairement délimitées et démarquées (statut des frontières). Si l'une de ces conditions seulement est remplie, le potentiel est considéré comme moyen alors que l'absence de délimitation ou de démarcation est considérée comme un signe de faible potentiel. En matière de pauvreté, le potentiel de coopération est maximum lorsque les inégalités entre régions frontalières ne sont ni trop grandes, ni trop petites, ce qui réduirait les possibilités ou l'incitation à coopérer. L'attribution de scores standardisés permet ensuite de mettre en évidence les zones potentiellement les plus favorables à la coopération. 
Tableau 2

Potentiel de coopération transfrontalière

\begin{tabular}{|c|c|c|c|}
\hline & & Potentiel & \\
\hline Indicateurs & $\begin{array}{c}\text { Élevé } \\
3 \text { points }\end{array}$ & $\begin{array}{l}2 \text { points } \\
\text { Moyen }\end{array}$ & $\begin{array}{l}1 \text { point } \\
\text { Faible }\end{array}$ \\
\hline Potentiel de population & $\begin{array}{l}\text { Nombre de personnes } \\
\text { atteignables en } \\
0-120 \text { minutes }\end{array}$ & $\begin{array}{l}\text { Nombre de personnes } \\
\text { atteignables en } \\
121-240 \text { minutes }\end{array}$ & \\
\hline Ressources en eau & $\begin{array}{l}\text { Eaux de surface et } \\
\text { souterraines partagées }\end{array}$ & $\begin{array}{l}\text { Eaux de surface ou souter- } \\
\text { raines partagées }\end{array}$ & \\
\hline $\begin{array}{l}\text { Ressources agricoles } \\
\text { et pastorales }\end{array}$ & $\begin{array}{l}\text { Bassins agricoles et } \\
\text { itinéraires pastoraux } \\
\text { partagés }\end{array}$ & $\begin{array}{l}\text { Bassins agricoles ou } \\
\text { itinéraires pastoraux partagés }\end{array}$ & $\begin{array}{l}\text { Absence de bassins } \\
\text { agricoles et itinéraires } \\
\text { pastoraux partagés }\end{array}$ \\
\hline Langues & $\begin{array}{l}\text { Absence de discontinuités } \\
\text { entre langues vernaculaires, } \\
\text { véhiculaires et coloniales }\end{array}$ & $\begin{array}{l}\text { Quelques discontinuités entre } \\
\text { langues vernaculaires, } \\
\text { véhiculaires et coloniales }\end{array}$ & $\begin{array}{l}\text { Fortes discontinuités } \\
\text { entre langues } \\
\text { vernaculaires, véhiculaires } \\
\text { et coloniales }\end{array}$ \\
\hline Statut des frontières & $\begin{array}{l}\text { Frontières clairement } \\
\text { délimitées et démarquées }\end{array}$ & $\begin{array}{l}\text { Frontières clairement } \\
\text { délimitées ou démarquées }\end{array}$ & $\begin{array}{l}\text { Frontières ni délimitées ni } \\
\text { démarquées }\end{array}$ \\
\hline Stabilité politique & $\begin{array}{l}\text { Absence de conflits } \\
\text { frontaliers et de groupes } \\
\text { transnationaux }\end{array}$ & $\begin{array}{l}\text { Instabilité politique occasion- } \\
\text { nelle en région frontalière }\end{array}$ & $\begin{array}{l}\text { Conflits frontaliers ou } \\
\text { groupes transnationaux } \\
\text { majeurs }\end{array}$ \\
\hline Pauvreté & $\begin{array}{l}\text { Différentiels moyens } \\
(10.4-24.0)\end{array}$ & & $\begin{array}{l}\text { Différentiels très petits ou } \\
\text { très grands }(0-10.3 \text { et } \\
24.1-100)\end{array}$ \\
\hline
\end{tabular}

Source : OCDE/CSAO 2017

La cartographie du potentiel de coopération met en évidence de fortes disparités entre régions divisées par une frontière terrestre en Afrique de l'Ouest et du Nord (Carte 1).

$\underline{\text { Carte } 1}$

Potentiel de coopération transfrontalière

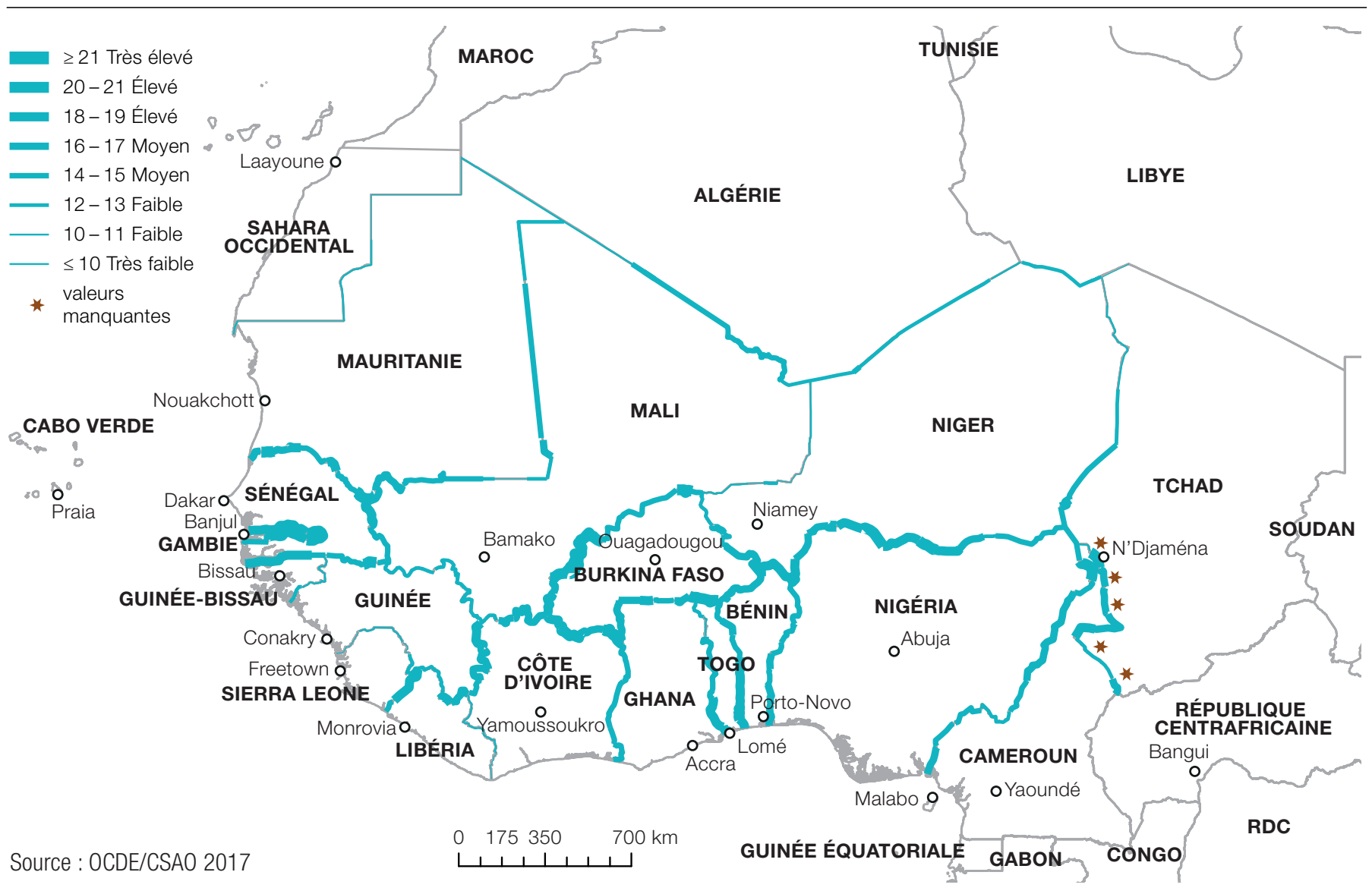


- Les espaces sahélo-sahariens sont globalement ceux qui jouissent du potentiel de coopération le plus faible. Cela s'explique surtout par la faiblesse du peuplement (Carte 2) et des ressources agricoles et par l'instabilité politique, en particulier au Mali, depuis le début des années 2000 avec l'arrivée d'extrémistes religieux et les revendications indépendantistes.

- Le Sahel est globalement caractérisé par de hauts potentiels de coopération, comme en Sénégambie méridionale, sur les frontières du Burkina Faso et entre le Niger et le Nigéria. Ces régions dotées de nombreux marchés frontaliers sont densément peuplées et partagent des ressources hydrographiques, agricoles et pastorales qui favorisent la mise en œuvre de filières de production et de commercialisation transfrontalières. Elles sont en outre peu fragmentées du point de vue linguistique, relativement épargnées par l'instabilité politique et les écarts de pauvreté y sont moyens. Du point de vue institutionnel, la présence de frontières reconnues par les États et démarquées sur le terrain y facilite la coopération transfrontalière.

- Les régions frontalières du Golfe de Guinée sont plus hétérogènes. Alors que de nombreux segments de frontières en Sierra Leone, en Guinée et au Libéria paraissent peu favorables à la coopération transfrontalière en raison de leur faible densité de marchés frontaliers, du statut incertain de leurs frontières et du relatif manque de ressources partagées, certaines régions présentent des valeurs très élevées, comme entre le Ghana, le Togo et le Bénin. La conurbation Accra-Lagos apparaît de ce point de vue particulièrement favorable à la coopération transfrontalière.

Carte 2

Potentiel de population des marchés frontaliers

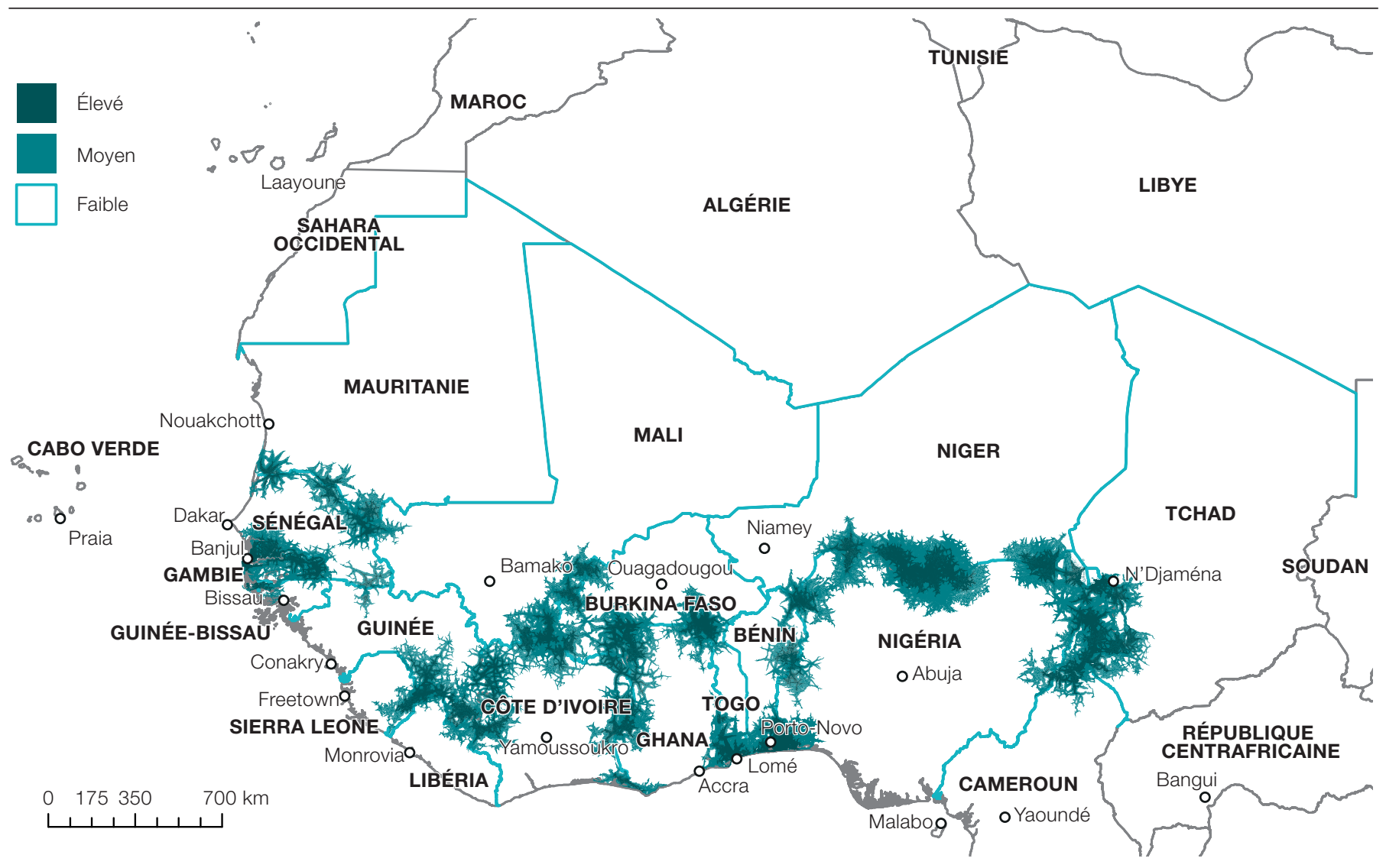

Source :OCDE/CSA0 2017 
Le calcul du potentiel de coopération constitue une première étape, indispensable mais non suffisante, à l'étude de la géographie de la coopération transfrontalière en Afrique de l'Ouest. L'intensité de celle-ci ne peut, en effet, pas seulement être mesurée au potentiel de ses régions, qui peut être ou non exploité par les acteurs socio-économiques et politiques. Afin de montrer comment les frontières actuelles peuvent servir de ressources au processus d'intégration régionale, encore faut-il analyser les réseaux de gouvernance de la région.

\section{La structure actuelle : où la coopération transfrontalière se développe-t-elle ?}

L'analyse de la structure des réseaux de gouvernance permet d'identifier qui sont les acteurs engagés dans la coopération transfrontalière, de cartographier quelles sont leurs relations formelles et informelles et d'évaluer l'impact des frontières nationales sur les échanges d'information et de pouvoir. Elle s'appuie sur une enquête inédite conduite auprès de 137 acteurs impliqués dans la coopération transfrontalière à l'échelle de l'Afrique de l'Ouest toute entière et dans trois microrégions : la vallée du fleuve Sénégal, le Liptako-Gourma et la région du lac Tchad (Carte 3). Chacune de ces régions possède une organisation sectorielle chargée de promouvoir les échanges transfrontaliers : l'Organisation de mise en valeur du fleuve Sénégal (OMVS), l’Autorité de développement intégré de la région du Liptako-Gourma (ALG), et la Commission du bassin du lac Tchad (CBLT).

$\underline{\text { Carte } 3}$

Afrique de l'Ouest et microrégions étudiées

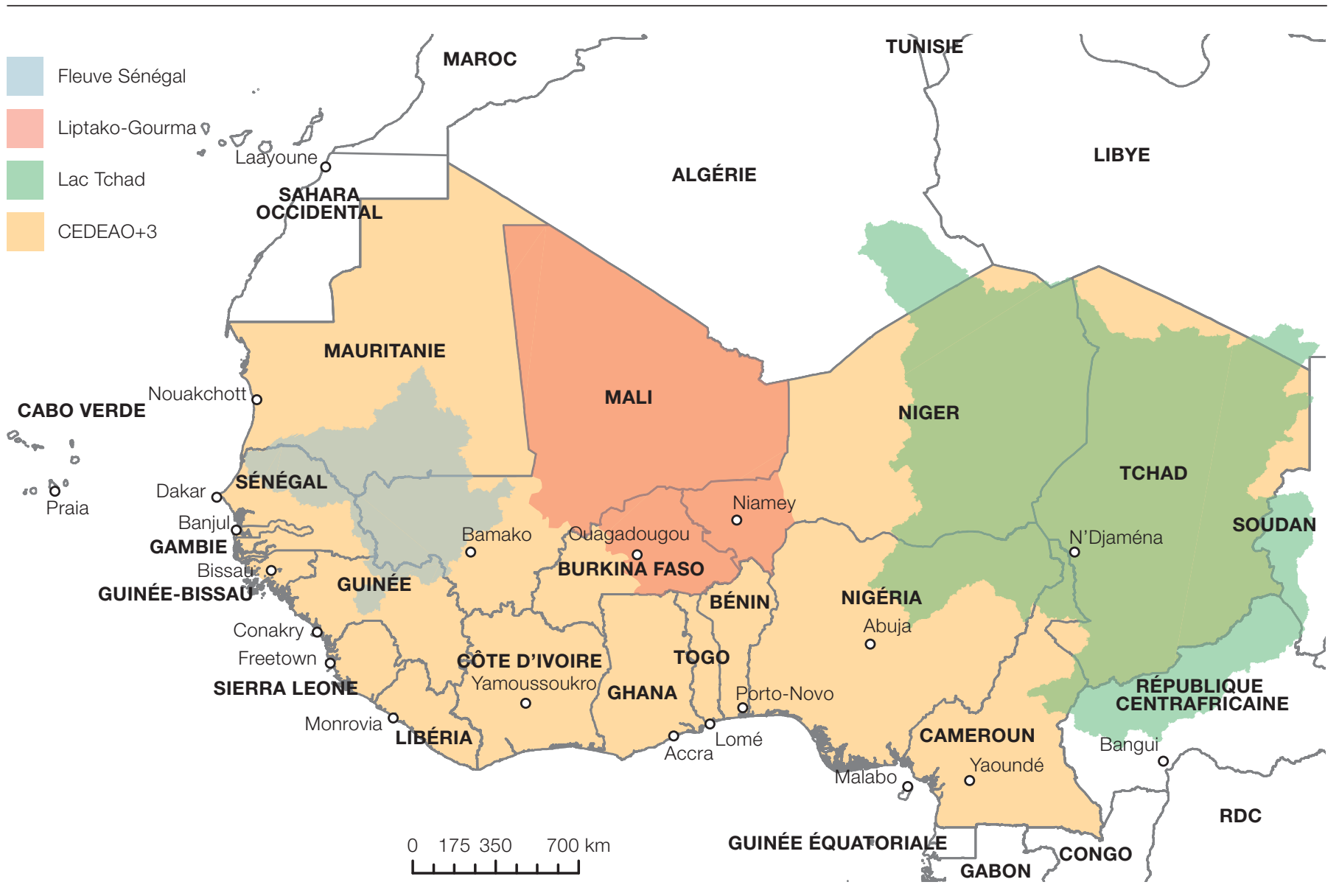

Source : OCDE/CSAO 2017 
Les réseaux de gouvernance sont reconstitués au moyen d'une analyse des réseaux sociaux (Social Network Analysis) qui focalise sur les liens entretenus entre acteurs d'un domaine d'activité spécifique. Plusieurs vagues d'entretiens menés en face-à-face auprès des acteurs impliqués dans la coopération frontalière permettent de cartographier les réseaux et de mesurer la centralité de chaque acteur. Les acteurs interviewés sont tout d'abord invités à nommer les personnes avec lesquelles ils ont personnellement échangé des informations relatives à la coopération transfrontalière de 2013 à 2015. Ceci permet de reconstituer le réseau d'information qui lie les acteurs de la coopération transfrontalière. Les acteurs interviewés sont ensuite amenés à nommer les acteurs qu'ils considèrent comme les plus importants dans le domaine de la coopération transfrontalière en Afrique de l'Ouest ou dans chacune des trois microrégions considérées. Cette information permet de reconstituer le réseau de pouvoir qui relie les acteurs de la coopération transfrontalière au cours des deux dernières années.

À l'échelle de l'Afrique de l'Ouest, l'analyse des échanges d'information et des relations de pouvoir montre que les réseaux de gouvernance sont dominés par les organisations intergouvernementales, comme l'UA, la CEDEAO, l'UEMOA ou le Comité permanent inter-États de lutte contre la sécheresse dans le Sahel (CILSS), qui représentent près de $70 \%$ des acteurs (Graphique 1). C'est au sein de ces organisations que les relations sont les plus fréquentes, représentant plus d'un tiers des liens entretenus en matière de coopération transfrontalière.

Graphique 1

Composition des réseaux par type d'acteurs

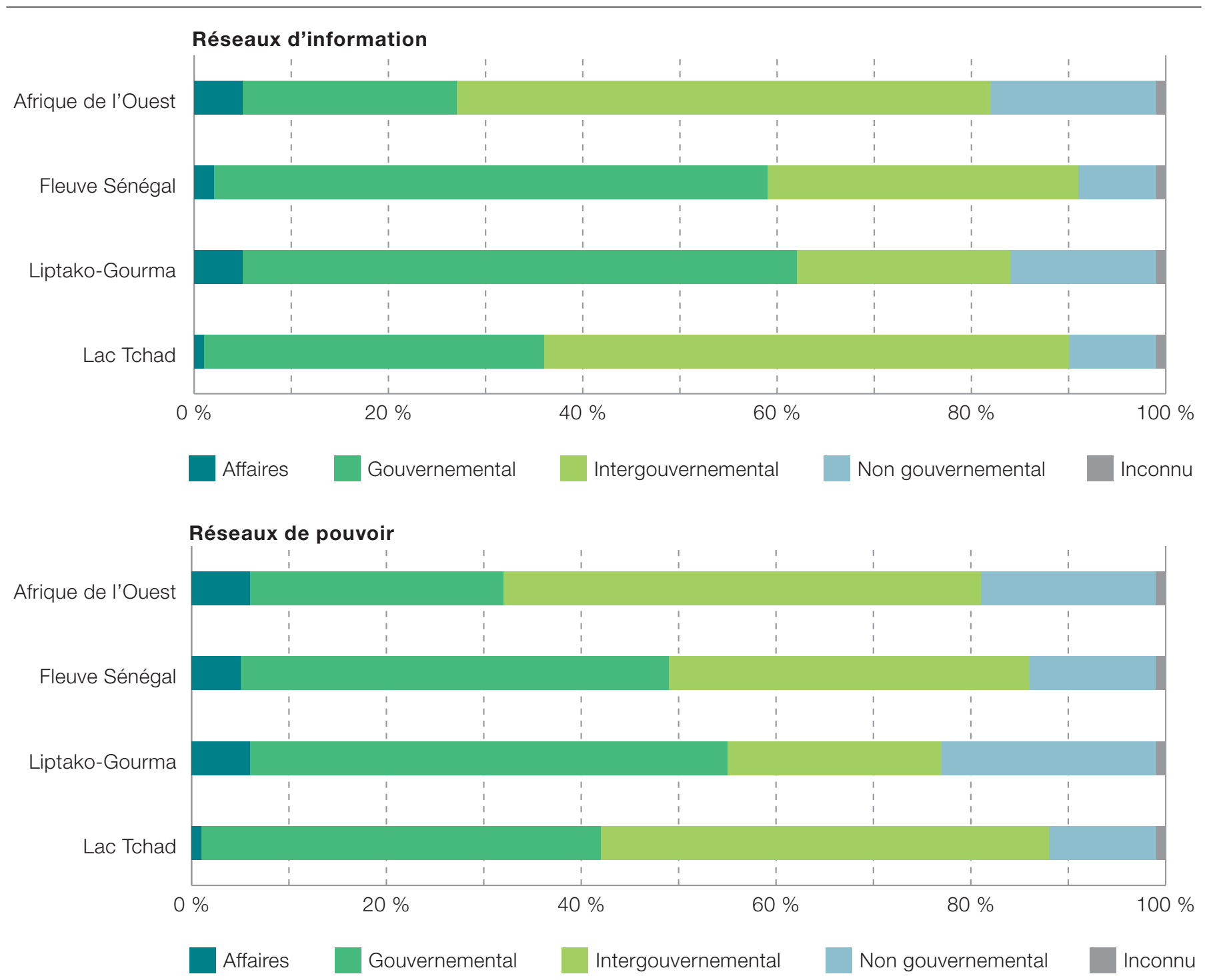


Les réseaux des microrégions sont caractérisés par une forte proportion d'organisations gouvernementales, du fait de l'importance des directions nationales impliquées dans les questions hydrauliques, environnementales et agricoles au sein de l'OMVS, de l'ALG et de la CBLT, pour lesquelles la plupart des acteurs les plus centraux travaillent.

- Dans la vallée du fleuve Sénégal, un quart des relations entretenues dans le réseau d'information sont internes aux organisations intergouvernementales tandis que le réseau de pouvoir est principalement structuré par des relations internes aux organisations gouvernementales (un tiers des liens).

- Dans le Liptako-Gourma, un tiers des relations d'information et de pouvoir sont internes aux différentes organisations gouvernementales actives dans la coopération transfrontalière entre Niger, Mali et Burkina Faso.

- Autour du lac Tchad, les organisations intergouvernementales dominent le réseau d'information et de pouvoir, avec plus de la moitié des acteurs. Les relations au sein de ces organisations représentent plus d'un quart des relations.

L'analyse montre en outre que la structure du réseau d'information au niveau de l'Afrique de l'Ouest ressemble à celle mise en place par les acteurs locaux dans les trois microrégions considérées. De faible densité (peu de liens entre acteurs) et plutôt décentralisée, la structure de ces réseaux de gouvernance semble adaptée à la circulation d'informations entre partenaires aux statuts et aux compétences très divers. Une structure décentralisée offre également plus de souplesse aux acteurs de la coopération, qui opèrent dans un environnement institutionnel souvent non contraignant, qu'une structure centralisée sur un seul pays ou une seule organisation.

Ces réseaux possèdent en outre de nombreux intermédiaires (brokers) sur lesquels repose la responsabilité de mettre en relation des sous-groupes qui entretiennent peu de relations directes, comme les représentants des éleveurs et les représentants des agences gouvernementales et intergouvernementales dans le Liptako-Gourma par exemple. Le rôle des brokers est particulièrement visible sur le Graphique 2, qui représente chaque acteur individuel en proportion de sa centralité d'intermédiarité à l'échelle de toute l'Afrique de l'Ouest. La centralité d'intermédiarité est une mesure de centralité globale qui indique si les acteurs sociaux établissent des liens entre des groupes autrement déconnectés. C'est au sein de la CEDEAO que les brokers les plus importants peuvent être observés : quatre représentants de cette institution figurent parmi les dix acteurs les plus centraux du réseau.

Quel rôle jouent les frontières dans ces réseaux? En s'appuyant sur le concept d'homophilie qui présume que les acteurs de la coopération transfrontalière tendent à privilégier les relations sociales avec d'autres acteurs partageant des attributs communs, comme la nationalité ou l'appartenance à une même organisation, l'analyse montre que les réseaux de gouvernance sont diversement affectés par les frontières.

À l'échelle régionale, ces réseaux apparaissent fortement structurés sur des bases nationales, notamment entre acteurs situés en Afrique et en Europe (« mesure entre appartenance au même continent )). Les réseaux régionaux présentent un taux d’homophilie élevé : plus de $67 \%$ des relations sont entretenues entre acteurs d'un même continent (Tableau 3). Ces résultats sont corroborés par le calcul de l'index E/I, qui mesure la différence entre les relations internes et externes de chaque pays ou continent, divisées par le nombre total de relations entretenues entre les acteurs d'un réseau. Cet indice est très clairement négatif et relativement élevé, confirmant l’homophilie des réseaux d’Afrique de l'Ouest. 
Graphique 2

Brokers du réseau d'information ouest-africain

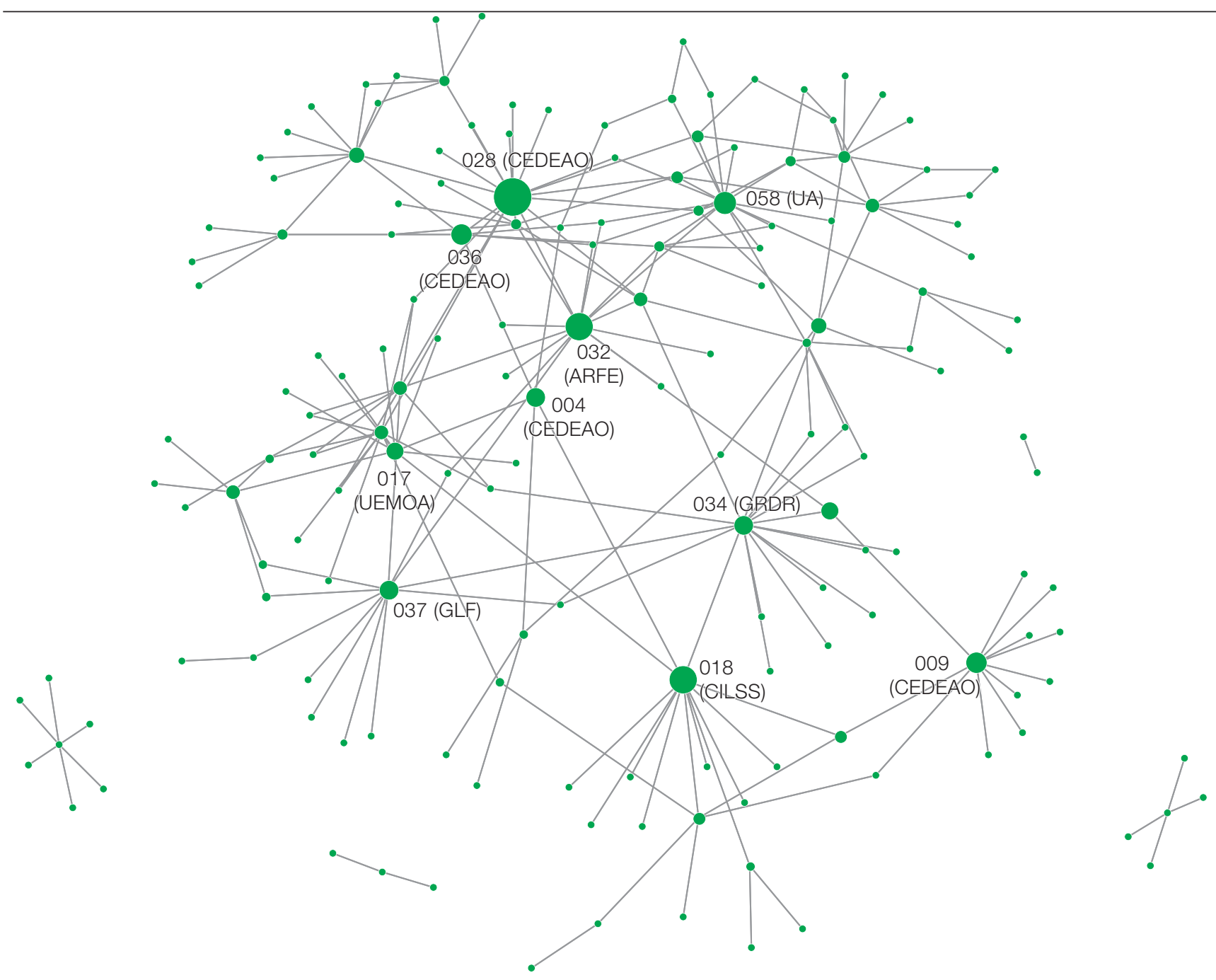

Source : OCDE/CSA0 2017. Note : seuls les codes et l'appartenance des dix acteurs les plus centraux sont représentés. Les acteurs impliqués dans la coopération transfrontalière sont représentés par des cercles proportionnels à leur rôle d'intermédiaire (broker) au sein du réseau. Acronymes : Communauté économique des États de l'Afrique de l'Ouest (CEDEA0), Union africaine (UA), Comité permanent inter-États de lutte contre la sécheresse dans le Sahel (CILSS), Union économique et monétaire ouest-africaine (UEMOA), Groupe de recherche et de réalisations pour le développement rural (GRDR), Association des régions frontalières européennes (ARFE), Global Local Forum (GLF).

L'effet des frontières sur les réseaux est atténué à l'échelle des trois microrégions considérées où les initiatives institutionnelles destinées à favoriser l'émergence de régions transfrontalières conduisent les acteurs à communiquer davantage avec des partenaires d'autres pays. Dans le Liptako-Gourma, par exemple, les faibles valeurs d'homophilie, comprises entre 29 et 35 \% , indiquent que les acteurs possèdent un réseau diversifié de partenaires dans plusieurs pays et ne favorisent pas systématiquement leur propre pays, ce qui est un signe de maturité de la coopération transfrontalière. Dans le réseau de pouvoir, les acteurs maliens (en vert) sont par exemple bien connectés aux acteurs burkinabés (en bleu) (Graphique 3). Les acteurs nigériens (en marron) occupent également une place d'importance, du fait de leurs nombreuses connexions et de leur rôle d'intermédiaire entre les autres acteurs du réseau (en beige). 
$\underline{\text { Tableau } 3}$

Homophilie par région

Réseau d'information

\begin{tabular}{lcccc}
\hline & Homophilie (\%) & Index E/I & Homophilie (\%) & Index E/I \\
\hline Afrique de l'Ouest & 67.5 & -0.350 & 67.6 & -0.352 \\
\hline Fleuve Sénégal & 28.8 & $0.424^{* *}$ & 36.4 & $0.273^{\star *}$ \\
\hline Liptako-Gourma & 34.8 & $0.303^{\star *}$ & 29.1 & $0.419^{\star}$ \\
\hline Lac Tchad & 21.0 & $0.581^{*}$ & 20.0 & 0.599 \\
\hline
\end{tabular}

Source : OCDE/CSA0 2017. Notes : L'index E/I varie entre 1 et -1. Une valeur fortement négative de l'index indique que le réseau est très homophile (les acteurs échangent principalement dans leur propre pays), tandis qu'une valeur fortement positive indique que le réseau est très hétérophile (les acteurs échangent sans tenir compte des frontières). Deux étoiles ( ${ }^{* \star}$ ) indiquent que l'index E/l est significatif à $5 \%$, $(p<0.05)$, une étoile $\left(^{*}\right)$ indique qu'il est significatif à $10 \%(p<0.1)$.

Graphique 3

Réseau d'information dans la région du Liptako-Gourma par pays

Burkina Faso

Mali

Niger

Autres
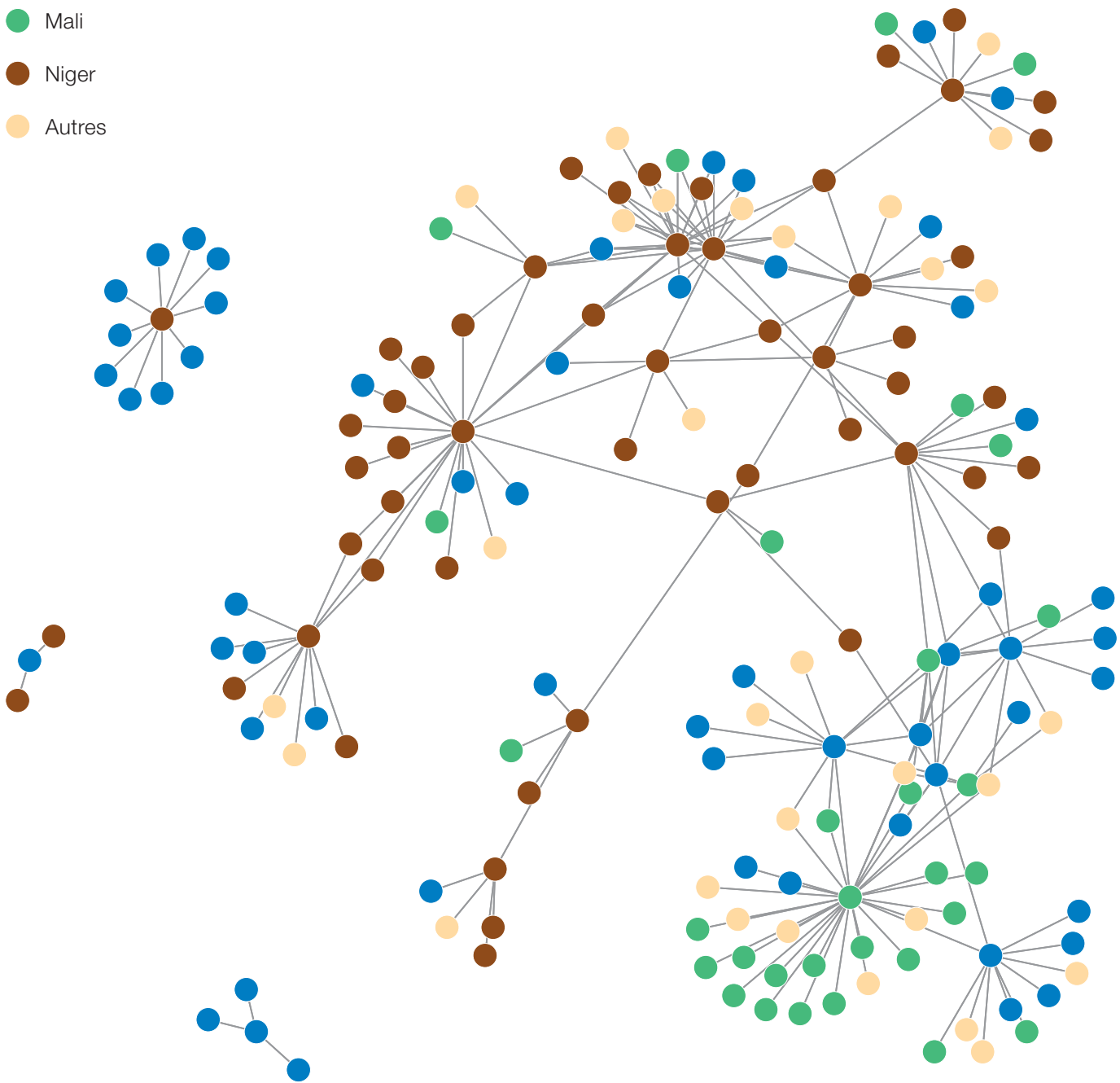

Source : OCDE/CSAO 2017 
La tendance à l'homophilie est également faible dans la région du lac Tchad, particulièrement pour les acteurs situés au Niger et au Nigéria, qui sont densément connectés à leurs homologues des pays voisins et cela quel que soit le type de réseau (information et pouvoir). Le réseau de gouvernance de cette région est ainsi caractérisé par des acteurs qui échangent au-delà des frontières mais principalement dans le cadre de leur structure - en l'occurrence la CBLT. Il diffère du réseau de la vallée du fleuve Sénégal et du Liptako-Gourma, où les acteurs de la coopération transfrontalière traversent plus volontiers les limites institutionnelles.

\section{La vision : où la coopération transfrontalière devrait-elle se développer ?}

L'une des questions les plus épineuses qui se pose aux acteurs impliqués dans les politiques publiques transfrontalières est de savoir où la coopération devrait se développer. Les régions frontalières doivent-t-elles s'appuyer sur les limites administratives existantes de chaque pays, sur des limites physiques plus difficilement contestables, comme les bassins hydrographiques, ou sur l'espace à géométrie variable défini par les interactions des populations qui traversent la frontière au quotidien ? Loin d'être anecdotique, la délimitation de l'espace d'action privilégié de la coopération transfrontalière détermine bien souvent quels seront les acteurs représentés au sein des structures de gouvernance, où seront concentrés les investissements et quels seront les rapports de pouvoir entre les régions frontalières et les autres niveaux administratifs.

L'espace d'action prioritaire de la coopération peut être reconstitué à partir des perceptions spatiales des acteurs impliqués dans la coopération transfrontalière. La construction régionale repose en effet sur une vision commune des espaces transfrontaliers entre partenaires de la coopération. L'objectif est ici de cartographier ces représentations spatiales, en identifiant les régions reconnues comme prioritaires pour la coopération transfrontalière en l'Afrique de l'Ouest, dans la région du fleuve Sénégal, du LiptakoGourma et du bassin du lac Tchad. Ce travail s'appuie sur une analyse inédite de 137 croquis cartographiques connus sous le nom de (c cartes mentales »), sur lesquelles chaque personne interviewée dessine les limites de ce qu'elle considère comme l'espace prioritaire de la coopération et indique où sont prises les décisions stratégiques en la matière (Carte 4).

La superposition de toutes les cartes mentales permet tout d'abord de vérifier s'il existe un consensus sur l'extension géographique de la coopération transfrontalière. Elles indiquent quelles sont les régions considérées comme prioritaires pour la coopération transfrontalière par une majorité d'acteurs et celles jugées plus secondaires. La surface moyenne des cartes mentales et leur densité par région sont utilisées pour juger de cette extension géographique (Carte 4). Les cartes mentales permettent ensuite de vérifier dans quelle mesure chaque carte mentale est plus ou moins dispersée dans l'espace par rapport au centre de gravité de toutes les cartes de la région. Le centre de gravité est plus important dans les microrégions qu'à l'échelle de l'Afrique de l'Ouest dans son ensemble, compte tenu de la très grande dimension de cette dernière, qui encourage la dispersion des cartes mentales.

L'analyse montre que les acteurs s'accordent rarement à la fois sur l'extension géographique et sur le centre de gravité géographique de la coopération transfrontalière (Tableau 4). À l'échelle régionale, il est difficile de parler de consensus sur l'extension géographique de la coopération, tant les cartes mentales diffèrent en taille selon les personnes interrogées. Tout au plus peut-on noter que les acteurs situés en Afrique de l'Ouest ont une vision plus moyenne de leur région que ceux situés en Europe ou dans le reste de l'Afrique. 
Carte 4

Cartes mentales à l'échelle locale

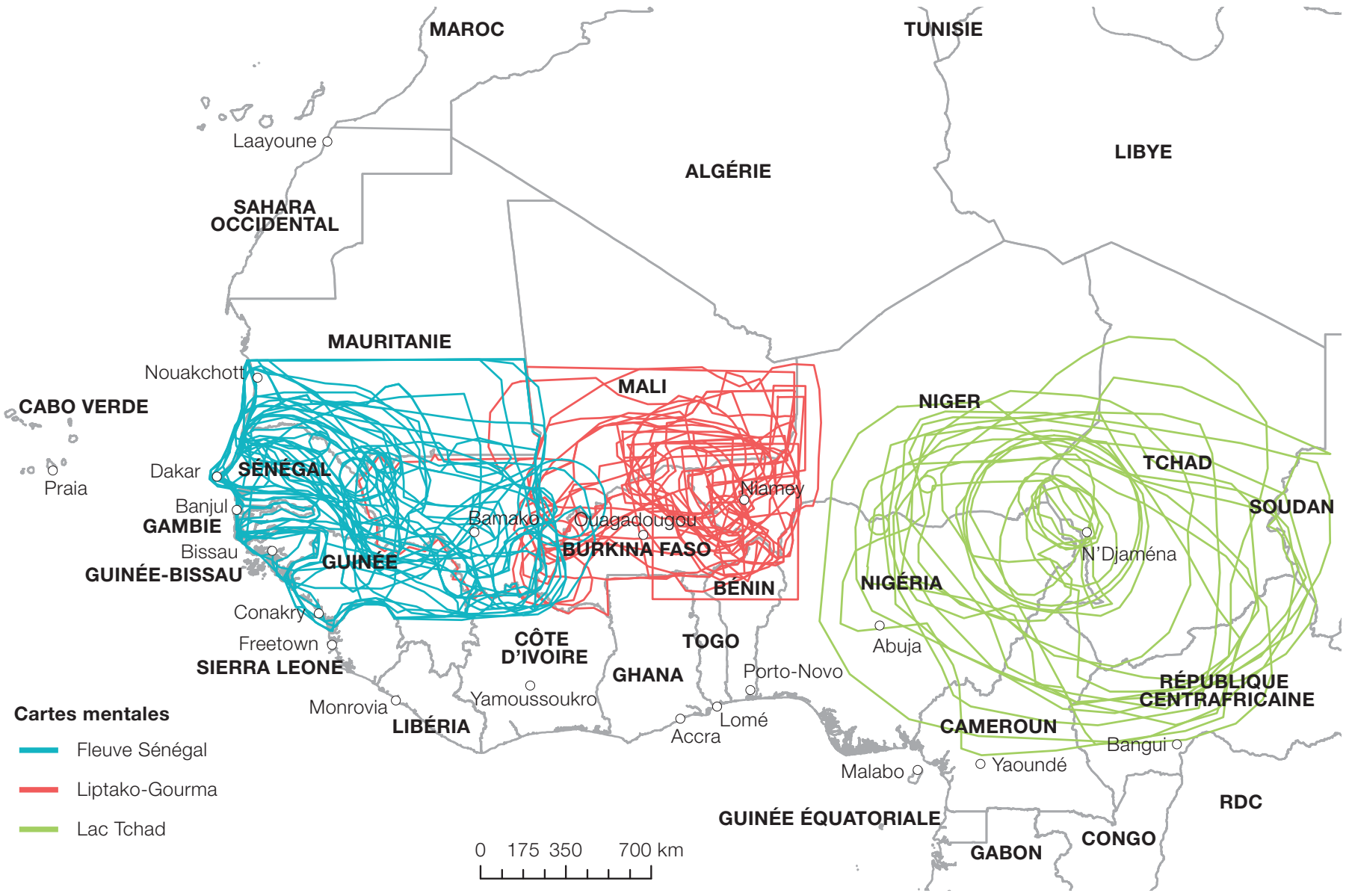

Source : OCDE/CSA0 2017

Tableau 4

Consensus sur l'extension et le centre de gravité de la coopération transfrontalière

\begin{tabular}{lcc}
\hline & Extension géographique & Centre(s) de gravité \\
\hline Afrique de l'Ouest & Non & Oui \\
\hline Vallée du fleuve Sénégal & Oui & Non \\
\hline Liptako-Gourma & Oui & Oui \\
\hline Région du lac Tchad & Non & Oui \\
\hline
\end{tabular}

Source : OCDE/CSAO 2017 
$\underline{\text { Carte } 5}$

Espace d'action prioritaire de la coopération transfrontalière en Afrique de l'Ouest

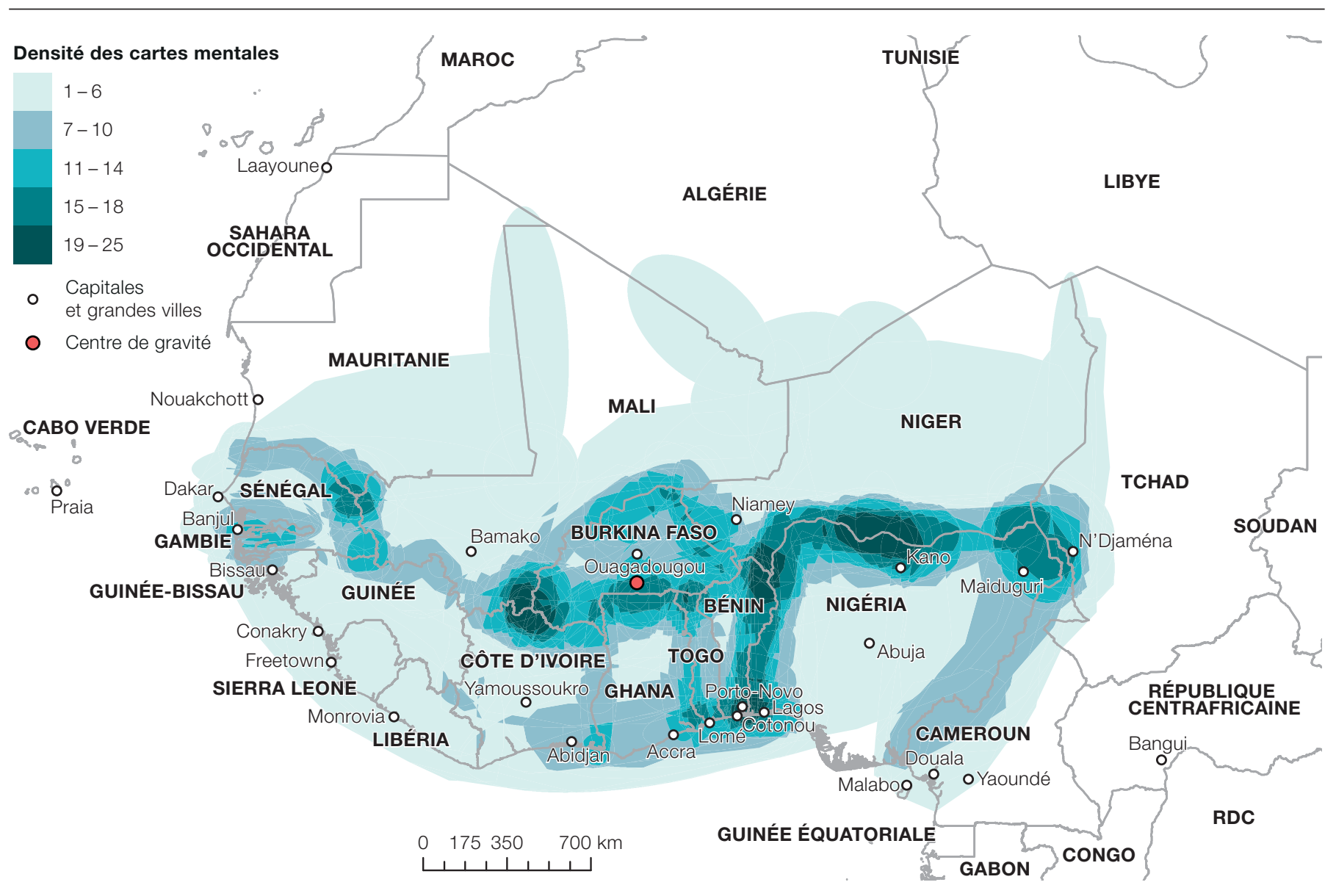

Source : OCDE/CSA0 2017

Plusieurs régions sont néanmoins reconnues comme des centres de gravité pour la coopération transfrontalière, comme la conurbation Lagos-Cotonou, le Dendi, le pays haoussa, la frontière entre le Togo et le Burkina Faso et le triangle Sikasso-Korhogo-Bobo Dioulasso (Carte 5). L'ouest et le nord du Nigéria, Lomé, le lac Tchad, le point triple entre Mali, Mauritanie et Sénégal et la frontière Burkina Faso-Ghana sont considérés comme plus secondaires tandis que la bande intermédiaire entre le Golfe de Guinée et le Sahel n'est pas perçue comme prioritaire pour la coopération transfrontalière.

- Dans la région du fleuve Sénégal, il existe un consensus sur l'extension géographique de la coopération transfrontalière, qui suit la vallée du fleuve et la frontière sénégalomalienne, mais non sur son centre de gravité, qui est fort variable. La partie reconnue comme prioritaire, qui s'étend sur 100000 km² environ, est presque trois fois plus réduite que le bassin du fleuve Sénégal et correspond à la partie sud du périmètre placé sous la compétence de l’OMVS.

- Dans le Liptako-Gourma, un consensus existe à la fois sur l'extension et le centre de gravité de la coopération transfrontalière. L'espace d'action privilégié de la coopération transfrontalière dessine un croissant incluant le point triple Burkina Faso-Niger-Mali et la vallée du fleuve Niger jusqu'à Niamey. Cette zone d'environ 50000 km² correspond au cœur du périmètre restreint de l'ALG, d'une superficie cependant près de sept fois et demie supérieure.

- Dans le bassin du lac Tchad, les acteurs s'accordent à reconnaître la zone N’DjaménaMaiduguri-Diffa comme le centre de la coopération transfrontalière mais ne s'entendent 
guère sur son étendue géographique, qui va pour certains acteurs jusqu'en République centrafricaine, au sud du Tchad et au centre du Niger. La zone reconnue comme la plus centrale, d'une superficie de 176000 km², représente un sixième de celle du bassin conventionnel du lac Tchad, sur lequel la CBLT exerce ses compétences.

Dans toutes les régions, l'espace d'action prioritaire de la coopération transfrontalière est nettement plus réduit que celui sur lequel s'appuient les compétences des organisations telles que l'OMVS, l'ALG et la CBLT (Carte 6). La grande taille des structures institutionnelles observée en Afrique de l'Ouest reflète un héritage historique marqué par la volonté de bâtir des régions à partir d'unités administratives existantes. Ce choix empêche cependant de concentrer les investissements transfrontaliers à une échelle plus réduite, par exemple sur les centres urbains des zones considérées comme les plus centrales, comme Kaédi, Sélibabi et Kayes, Dori, Gao et Tillabéri, ou N’Djaména, Maiduguri et Diffa.

Jusqu'ici, force est de constater que les petites et moyennes villes ouest-africaines ne figurent pas parmi les centres où se prennent les décisions stratégiques. L'enquête conduite auprès des 137 acteurs de la région confirme en effet que les décisions politiques relatives à la coopération restent prises dans les capitales de la région. Ces lieux stratégiques sont avant tout Abuja, au Nigéria, où siège la CEDEAO, Ouagadougou au Burkina Faso où siègent l'UEMOA et le CILSS et, dans une moindre mesure, Dakar, au Sénégal. À elles seules, ces trois capitales représentent près de 40 \% des lieux mentionnés au moins une fois comme stratégiques pour la coopération à l'échelle régionale par les personnes enquêtées.

Les capitales des États membres des organisations sectorielles figurent également parmi les lieux les plus stratégiques pour la coopération au niveau local, du fait qu'elles abritent les sièges des organisations sectorielles de coopération transfrontalière (Carte 7).

- C'est le cas dans la vallée du fleuve Sénégal, où Dakar, Bamako, Nouakchott et Conakry ressortent très largement comme les lieux principaux où se prennent les décisions stratégiques du fait de la présidence tournante de l'OMVS. Ces lieux incontournables distancent nettement les capitales plus lointaines du Niger et du Burkina Faso, ainsi que les centres régionaux comme Kayes, Sikasso et Ségou, au Mali.

- Dans le Liptako-Gourma, Ouagadougou est très largement considérée comme le lieu le plus stratégique pour la coopération du fait qu'elle accueille l'UEMOA et l'ALG. Les autres capitales des pays membres de l'ALG - Niamey et Bamako - sont également considérées comme particulièrement centrales. Parmi les lieux les plus mentionnés figure la petite ville de Dori au Burkina Faso, dont l'importance stratégique est sans commune mesure avec sa taille démographique, du fait des initiatives qui y ont été prises au cours des dernières années dans le domaine de la coopération décentralisée. Le Liptako-Gourma apparaît comme un espace dont le potentiel est mis à profit par des réseaux de gouvernance transfrontaliers, en particulier le long de la frontière entre le Burkina Faso et le Niger. Cette région est aussi caractérisée par de nombreuses initiatives locales et un réseau dense d'acteurs situés dans de nombreux centres de décision de petite et moyenne tailles. Ce polycentrisme, conjugué à un réel dynamisme des communes, peut être considéré comme un avantage dans la mise en œuvre de programmes d’intégration « par le bas »).

- Les décisions stratégiques en matière de coopération transfrontalière dans la région du lac Tchad sont prioritairement prises dans la capitale tchadienne, puis dans les capitales des pays voisins. Certes, la région accueille la plus ancienne organisation de bassin d'Afrique, mais elle reste peu connectée aux réseaux de gouvernance régionaux. À l'échelle locale, la polarisation du réseau de gouvernance sur les acteurs de la CBLT, ne contribue guère à valoriser les espaces frontaliers, notamment ceux entre le Cameroun et le Tchad. 
Carte 6

Espace d'action prioritaire de la coopération transfrontalière dans les micro-régions
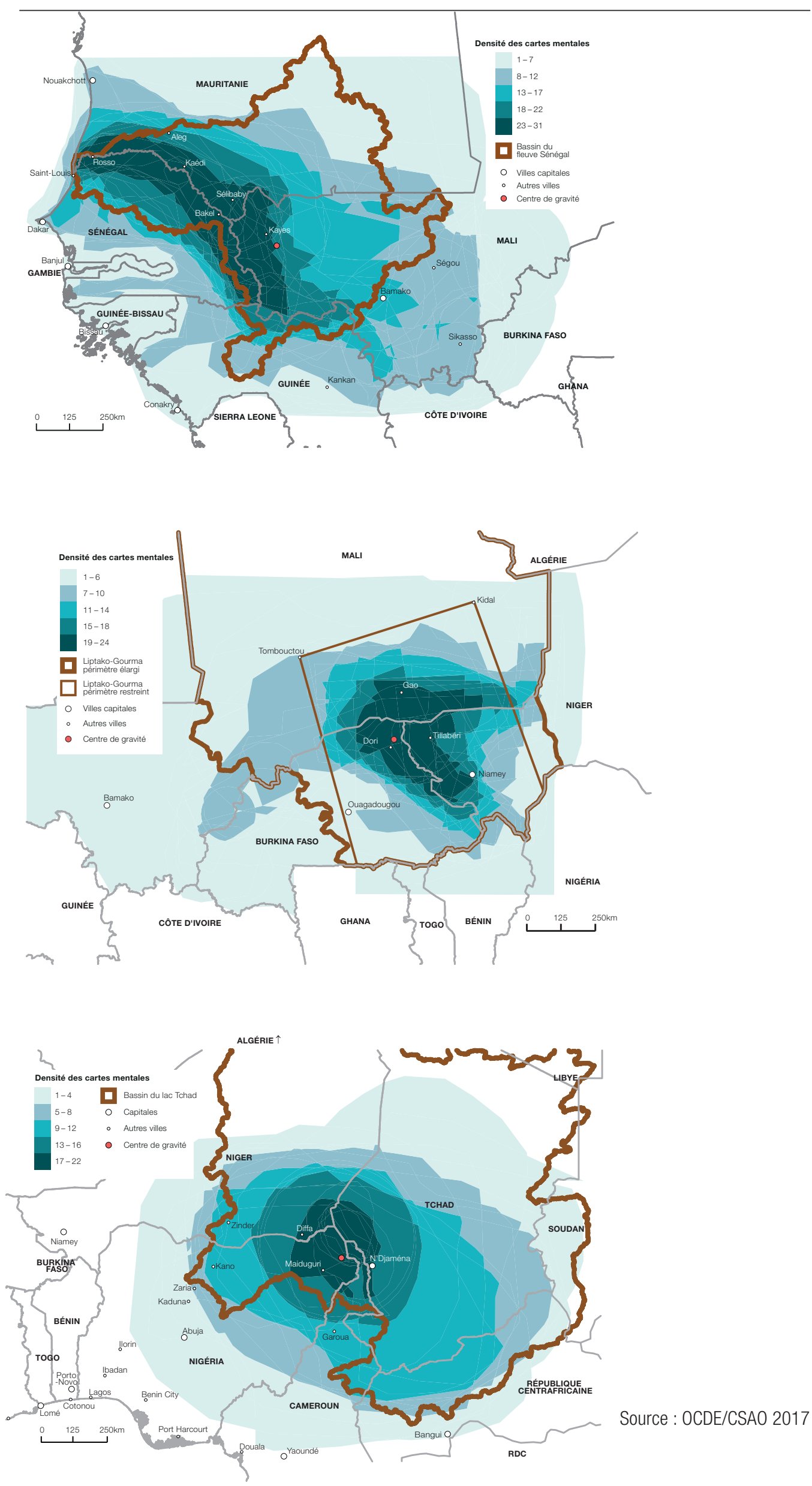
Carte 7

Lieux stratégiques pour la coopération transfrontalière à l'échelle locale

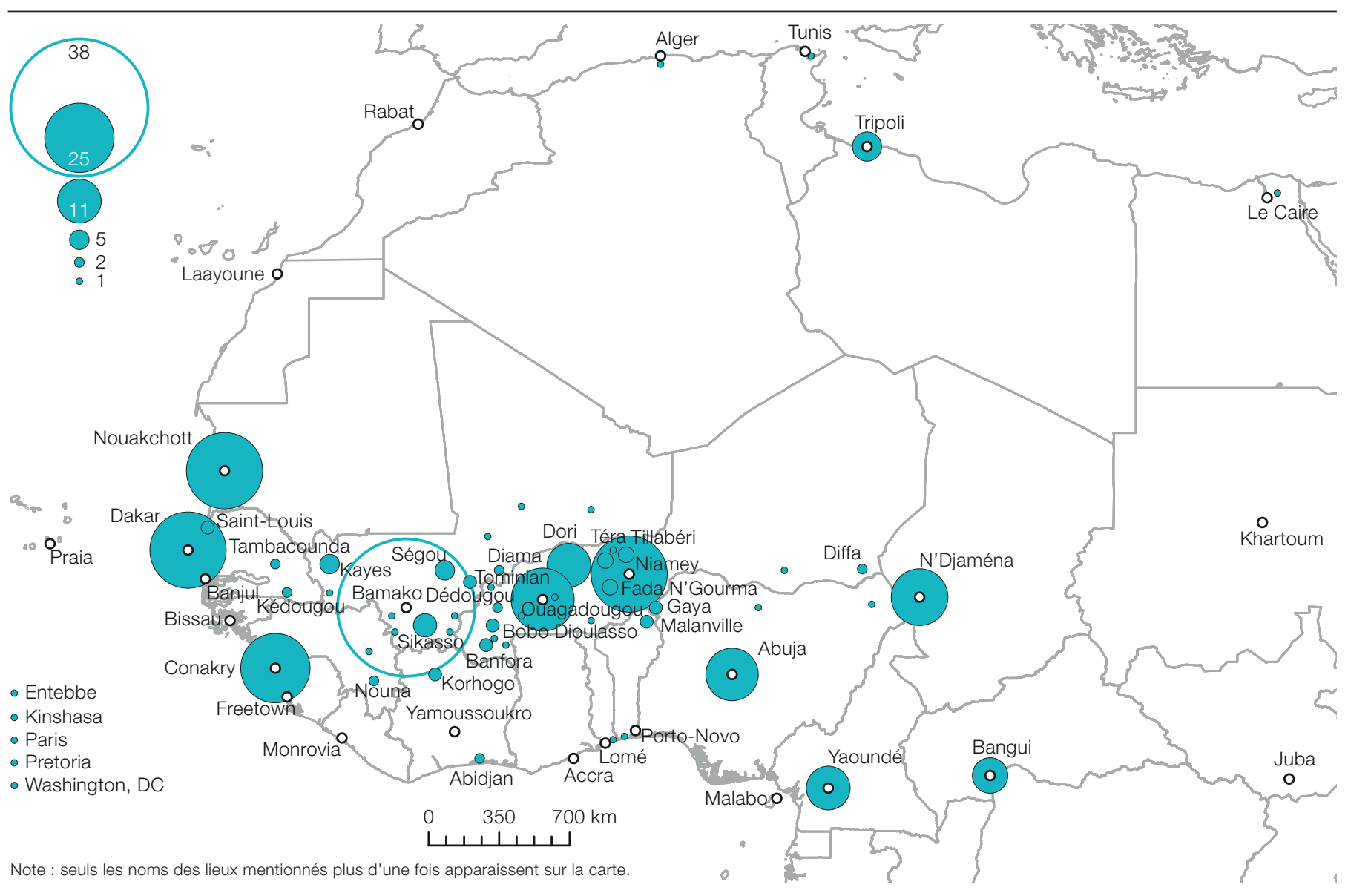

Source : OCDE/CSAO 2017

\section{CONJUGUER LES TROIS DIMENSIONS DE LA COOPÉRATION TRANSFRONTALIÈRE}

es politiques de coopération transfrontalière peuvent être considérées comme pleinement intégrées si elles se basent sur un potentiel avéré d'intégration, sur un réseau de gouvernance fonctionnel, et sur une vision partagée par les décideurs politiques. Cette situation idéale caractérise un petit nombre de régions, parmi lesquelles l'ouest du Mali, le triangle Sikasso-Korhogo-Bobo Dioulasso (SKBo), le Liptako-Gourma, le Dendi, la conurbation Lagos-Accra et le nord du Ghana. Dans le reste de l'Afrique de l'Ouest, de fortes contraintes politiques, institutionnelles et financières s'exercent sur la conception et l'implantation des politiques transfrontalières et expliquent qu'il existe un écart entre potentiel des régions, gouvernance et vision politique. Trois cas de figures principaux peuvent être observés.

\section{Un potentiel de coopération inexploité}

Le premier type de situation concerne les régions où la coopération trouve des conditions potentiellement favorables, sans toutefois que les acteurs locaux soient particulièrement bien reliés aux réseaux de gouvernance régionaux. Les frontières de Gambie, de Sierra Leone et du Libéria, sont représentatives de ce genre de situation, qui témoigne d'un potentiel inexploité. Bien que chacune de ces régions possède un organisme intergouvernemental potentiellement capable d'animer la coopération transfrontalière au niveau local - la Commission du bassin du fleuve Gambie et l'Union du fleuve Mano - leur inscription dans les réseaux ouest-africains demeure marginale. Les capitales et grandes villes de ces quatre pays n'accueillent pas d'acteurs particulièrement centraux dans la coopération ou d’institutions régionales comparables à celles que l'on trouve à Ouagadougou ou Abuja. 


\section{Des réseaux de gouvernance peu développés}

Le second type de situation concerne les régions reconnues comme prioritaires pour la coopération transfrontalière par les décideurs politiques, mais où les réseaux de gouvernance locaux et régionaux sont peu développés. Un écart important entre vision politique et gouvernance transfrontalière effective peut résulter d'un manque d'initiative des acteurs locaux, d'une préférence pour les échanges interpersonnels conduits en dehors des structures de gouvernance formelles, d'un dysfonctionnement dans l'échange d'information entre pays frontaliers ou d'une inclinaison à privilégier les échanges entre acteurs d'un même pays plutôt que les relations transfrontalières. Dans toutes ces situations, l'étude de la structure même des réseaux de gouvernance, par le biais de l'analyse des réseaux sociaux, permet de révéler les éventuels blocages institutionnels et de mettre en évidence les potentialités encore inexploitées de tisser un réseau dépassant les frontières nationales.

\section{Des priorités politiques}

Les régions où la coopération transfrontalière trouverait des conditions potentiellement favorables, sans toutefois être considérées comme prioritaires, résultent d'un arbitrage politique. Certaines régions comme la frontière nord entre Togo et Bénin et la Sénégambie méridionale pourraient en effet être favorables à la coopération mais leur développement n'est pas reconnu politiquement du fait des rapports de force entre élites nationales et collectivités régionales et locales. Dans les États centralisés, les investissements sont généralement concentrés dans un petit nombre de régions, en particulier celles des capitales, aussi longtemps que la décentralisation ne s'accompagne pas d'un transfert effectif de ressources et de pouvoir vers les collectivités locales et régionales. Pour les États fédéraux ou ayant conduit une réelle décentralisation, la question n'est pas tant de savoir si les investissements devraient être plus également distribués géographiquement, que de fixer des priorités quant à ceux qui pourront effectivement être réalisés compte tenu des finances publiques. C'est le cas de l'est du Nigéria, où le haut potentiel de coopération ne se traduit pas en priorité politique, dans la mesure où l'essentiel des ressources attribuées à la coopération par l'État fédéral et ses régions est concentré autour du lac Tchad.

\section{LE FUTUR DE LA COOPÉRATION TRANSFRONTALIÈRE}

I es politiques publiques mises en œuvre par les organisations régionales, les États — ouest-africains et leurs partenaires étrangers peuvent contribuer à promouvoir la coopération transfrontalière en adoptant des politiques territorialisées adaptées aux spécificités de chaque région et en développant un modèle d'intégration plus spécifiquement ouest-africain.

\section{Adapter les politiques aux spécificités des régions}

La plupart des politiques mises en œuvre en Afrique de l'Ouest promeuvent certains secteurs comme l'industrie et l'élevage ou certaines catégories de la population comme les plus vulnérables, sans nécessairement tenir compte des dimensions spatiales du développement régional. En conséquence, les disparités régionales sont en grande partie négligées, malgré le rôle critique qu'elles jouent au détriment de la croissance inclusive (BAD/OCDE/PNUD, 2015). Les politiques favorisant la coopération transfrontalière doivent rompre avec ce schéma et s'appuyer sur la grande diversité des régions d'Afrique de l'Ouest. Si elles veulent être efficaces, ces politiques doivent devenir des politiques territorialisées. Elles doivent tenir compte de la variété des besoins, des systèmes institutionnels et des niveaux de développement des régions ouest-africaines et fournir des biens publics adaptés aux problèmes socio-économiques spécifiques de chaque région. Des régions densément peuplées, comme le pays haoussa entre Niger et Nigéria ou le bassin du lac Tchad par exemple, ont des besoins différents de ceux des régions faiblement peuplées comme le Hodh à la frontière de la Mauritanie et du Mali. Les bandes côtières et plus industrialisées, comme la conurbation Accra-Lagos, requièrent des politiques sans grande utilité pour les régions agricoles comme au nord du Ghana. 
Ces stratégies territorialisées partent du principe que les acteurs et les institutions locaux peuvent être mobilisés pour soutenir le développement régional (OCDE, 2009 ; Barca et al., 2012). À la différence des politiques régionales classiques qui s’appuient sur des interventions sectorielles, ( descendantes ) et faisant appel aux subventions, les politiques territorialisées visent à promouvoir l'intégration spatiale en investissant dans les infrastructures et les biens publics adaptés aux contextes de chaque zone. Ces politiques sont fondées sur l'idée que les institutions et les acteurs locaux déterminent le potentiel de développement de la coopération transfrontalière et peuvent être mobilisés pour stimuler le développement économique. Elles s'adaptent aux conditions locales dans lesquelles le régionalisme s'inscrit, de manière à ce que les 32000 km de frontières ouest-africaines puissent favoriser des initiatives de coopération transfrontalière spécifiques.

La forte hétérogénéité des espaces frontaliers ouest-africains suggère que les investissements soient concentrés dans les régions qui possèdent le plus haut potentiel, ont déjà mis en œuvre des réseaux de gouvernance ou sont reconnues comme prioritaires par les décideurs politiques. Dans les autres régions où il existe un potentiel de coopération inexploité ou un consensus politique sur l'importance de la coopération frontalière mais où les réseaux de gouvernance sont encore peu développés, l'objectif des politiques territorialisées est de favoriser l'émergence de réseaux transfrontaliers d'acteurs. Cela peut être achevé en favorisant la création d'institutions appropriées, en réduisant les trop grands différentiels frontaliers qui limitent les possibilités de collaboration, en prévenant les conflits locaux ou internationaux ou en favorisant la connaissance réciproque des acteurs situés dans le pays voisin.

Le cas des régions possédant un fort potentiel de coopération mais non reconnue comme prioritaires par les décideurs politiques présente les plus fortes contraintes en termes de développement transfrontalier. Cela s'explique par le fait que la construction de microrégions suppose que les autorités locales et régionales soient suffisamment autonomes en ressources et en pouvoir pour faire valoir leurs intérêts auprès du gouvernement central. En l'absence de régions institutionnellement fortes, il est en effet difficile de parler de construction régionale. Dans ce type de région, l'exploitation du potentiel de coopération nécessite que soient mis en avant les effets positifs liés à un accroissement des interactions transfrontalières.

\section{Quel modèle d'intégration entre institutions et interactions fonctionnelles ?}

La vision politique de la coopération transfrontalière véhiculée en Afrique de l'Ouest emprunte aux deux grands modèles d'intégration régionale développés dans le monde, sans toutefois parvenir à formuler un modèle adapté aux spécificités africaines.

D'une part, l'expérience historique - et le soutien institutionnel et financier - de l'Union européenne (UE) incite les États ouest-africains à adopter un modèle d'intégration dans lequel la priorité est donnée aux structures institutionnelles. Ce modèle s'appuie sur la construction préalable de structures formelles de coopération transfrontalière, qui sont ensuite chargées de promouvoir le rapprochement entre les acteurs frontaliers et de favoriser les échanges socio-économiques. Les organisations régionales sectorielles mises en place depuis les années 60, comme l'OMVS, l'ALG et la CBLT, témoignent de ce modèle qui donne la priorité à la construction politique. L'intégration croissante entre régions frontalières est perçue comme une conséquence des efforts déployés par le cadre institutionnel.

D’autre part, ces dernières années ont vu le développement d'initiatives plus marquées et davantage aidées financièrement - par un modèle d'intégration donnant la priorité aux interactions entre acteurs socio-économiques, particulièrement répandu en Amérique du Nord. Ce modèle se base sur l'existence de complémentarités fonctionnelles entre territoires frontaliers pour mettre en place des structures de coopération ad hoc destinées avant tout à réduire les frictions qui entravent le commerce régional. La mise en place des postes de douane juxtaposés, la réhabilitation des corridors de transport et la levée des contrôles et pratiques informelles à l'origine d'une fragmentation de l'espace régional 
particulièrement onéreuse pour les opérateurs économiques transfrontaliers ouestafricains (USAID, 2015) illustrent cette orientation. Dans ce modèle, les structures de coopération transfrontalière sont au service des flux de biens et de personnes. Il en résulte des structures légères, parfois privées, impliquant autant les autorités publiques que les entreprises concernées par une thématique particulière, comme le transport ou l'environnement. Ces structures privilégient la mise en réseau des acteurs, quel que soit d'ailleurs leur échelon administratif.

La coexistence actuelle de ces deux modèles d'intégration en Afrique de l'Ouest permet de diversifier les formes données aux structures de la coopération transfrontalière et de bénéficier des financements qui sont liés à chacun d'eux. Dans un contexte marqué à la fois par une certaine prolifération d'organisations intergouvernementales et par une prépondérance des interactions informelles, il n'est pas certain qu'aucun de ces modèles ne soit réellement adapté aux opportunités et contraintes auxquelles font face les acteurs étatiques et non gouvernementaux en Afrique de l'Ouest.

Si le modèle fortement institutionnalisé d'inspiration européenne a l'avantage de créer des structures aisément identifiables pour les bailleurs internationaux, il multiplie les structures bureaucratiques. Dans la mesure où l'objectif principal de la coopération transfrontalière est de créer des ensembles infrarégionaux, ce modèle conduit naturellement à favoriser les représentants territoriaux comme les États, les régions et les communes, tandis que les acteurs non territoriaux et privés sont souvent absents. À l'inverse, si le modèle flexible d'inspiration nord-américaine a le mérite de concentrer ses actions sur un nombre restreint de problèmes fonctionnels, il se heurte souvent aux intérêts des États ouest-africains, pour lesquels la coopération transfrontalière est un champ politique comme un autre et les frictions ralentissant l'intégration régionale une source de revenus. À long terme, le succès de la coopération transfrontalière repose sur la mise en place d'un modèle d'intégration plus spécifiquement ouest-africain, c'est-à-dire qui intègre plus étroitement les spécificités socio-économiques et politiques de la région.

\section{APPRÉHENDER LA COOPÉRATION TRANSFRONTALIÈRE DE MANIÈRE RELATIONNELLE}

a coopération transfrontalière est un processus d'intégration qui devrait être plus _ systématiquement appréhendé de manière relationnelle, c'est-à-dire en considérant les interactions entretenues entre ses acteurs. Une attention exclusivement portée aux attributs institutionnels des organisations régionales, des États et des collectivités locales masque le fonctionnement réel de la coopération transfrontalière, dont l'efficacité repose bien souvent sur des relations interpersonnelles entre acteurs de nature très différente. L'ensemble de ces acteurs forme un réseau de gouvernance qui transcende à la fois les limites administratives des États et leurs propres divisions internes. Son fonctionnement échappe à toute simplification, du fait du grand nombre d'acteurs participant au processus d'échange d'information et de prise de décision. Dans la région de la vallée du fleuve Sénégal, par exemple, pas moins de 165 acteurs individuels, reliés par plus de 300 relations, sont directement impliqués dans la coopération transfrontalière.

Contrairement aux analyses fondées sur la constitution d'un échantillon d'individus statistiquement indépendant, l'analyse des réseaux sociaux cherche tout d'abord à reconstituer l'ensemble des relations entretenues au sein d'un réseau social. Elle tient alors compte de la position structurelle de chacun des acteurs, quel que soit son niveau hiérarchique, son appartenance nationale ou son autorité institutionnelle. 
Ce faisant, l'analyse des réseaux sociaux contribue à replacer l'acteur social au centre des études de développement, en le considérant comme un nœud indispensable à la cohésion de la structure sociale et non comme un individu statistiquement interchangeable. Plutôt que de se concentrer sur la collecte d'un grand nombre d'attributs, comme le genre, le niveau d'éducation ou le revenu, l'analyse des réseaux sociaux considère également les relations comme son unité d'analyse principale. Cette attention portée à l'étude des liens qui relient les acteurs sociaux est tout particulièrement adaptée à la coopération transfrontalière, dont l'objectif premier est de favoriser les interactions entre partenaires séparés par des frontières nationales.

À cet égard, l'analyse conduite en Afrique de l'Ouest suggère que la structure générale des réseaux de gouvernance conditionne fortement l'échange d'information et de pouvoir entre acteurs. Les réseaux décentralisés sont, à ce propos, particulièrement adaptés aux contraintes de la coopération transfrontalière, qui nécessite une coordination continue entre acteurs aux compétences très diverses. L'analyse de réseaux montre, en outre, que l'intégration régionale est facilitée, d'une part, par l'inscription des acteurs de la coopération dans des ensembles denses de partenaires (enracinement) et, d'autre part, par la construction de liens qui dépassent le niveau local (intermédiarité). Tandis que l'enracinement renforce la confiance entre acteurs proches, réduit les risques liés au montage des projets et favorise l'émergence d'une vision commune des régions frontalières, l'intermédiarité établie par les brokers leur permet d'accéder à des ressources nouvelles dans d'autres régions frontalières ou au niveau des organisations régionales.

L'analyse des réseaux sociaux constitue à la fois un outil de recherche pour les sciences sociales et économiques, un levier d'autonomisation pour les communautés locales et les organisations non gouvernementales ainsi qu'une aide à l'opérationnalisation pour les organisations internationales et les gouvernements. La possibilité de visualiser les relations effectivement entretenues au sein d'un groupe social permet ainsi aux communautés locales d'éclairer les causes structurelles de leur marginalisation. Ce genre d'approche participative a été conduit en Afrique subsaharienne et en Asie par plusieurs organisations internationales dans les domaines de l'agriculture, de la gestion des ressources naturelles et de la santé (Schiffer, 2012).

L'analyse de réseaux est aussi utile pour comprendre l'impact social des projets de développement sur les communautés locales (Banque mondiale, 2012). Qu'il s'agisse de l'adoption de nouvelles technologies agricoles, de la construction d'infrastructures hydrauliques ou de la mise en place d'un réseau de santé, l'approche par les réseaux facilite la mise en évidence de difficultés particulières qui entravent le développement des communautés, la visualisation de la complexité des acteurs impliqués dans la résolution des problèmes et la représentation des relations entre les difficultés elles-mêmes (Boutilier, 2011 ; Schiffer et al., 2012).

Encore peu appliqué au champ du développement, ce genre d'approche est donc un outil de recherche particulièrement adapté à la compréhension d’organisations sociales fluides, qui ne peuvent être aisément divisées en catégories prédéterminées, comme les décideurs impliqués dans la coopération transfrontalière. Elle pourrait trouver des applications nouvelles à d'autres champs relationnels par nature, comme le commerce, les migrations ou les conflits (Berrou et Combarnous, 2011 ; Walther, 2014, 2015), pour autant que des données relationnelles sur les relations d’affaires entre marchands, les échanges interpersonnels entre migrants, et les alliances ou affrontements entre groupes armés soient plus systématiquement collectées. 


\section{RÉFÉRENCES}

ARFE (2012), Possibilités de coopération transfrontalières en Afrique de l'Ouest : une contribution au processus d'intégration régionale, Association des régions frontalières européennes, Gronau.

BAD/OCDE/PNUD (2015), Perspectives économiques en Afrique 2015 : développement territorial et inclusion spatiale, Éditions OCDE, Paris, http://dx.doi.org/10.1787/aeo-2015-fr.

Banque mondiale (2012), Agricultural Innovation Systems: An Investment Sourcebook, Banque mondiale, Washington, DC.

Barca, F., P. McCann et A. Rodríguez-Pose (2012), "The Case for Regional Development Intervention: Place based Versus Place-neutral Approaches », Journal of Regional Science, vol. 52, n 1, pp. 134-152.

Berrou, J.P. et F. Combarnous (2011), "Testing Lin's social capital theory in an informal African urban economy ", The Journal of Development Studies, vol. 47, n 8, pp. 1216-1240.

Boutilier, R. (2011), A Stakeholder Approach to Issues Management, Business Expert Press, New York.

CSAO/OCDE (2007), Guide pratique de la coopération transfrontalière, CD-ROM, Paris, www.oecd.org/fr/csao/publications/guide-cooperation-transfrontaliere.htm.

Enda Diapol (dir. pub.) (2007), Les dynamiques transfrontalières en Afrique de l'Ouest, Textes réunis et présentés par Enda Diapol, Éditions Karthala, Paris.

Schiffer, E., A.Y. Mustapha et A.L. Mustaph (2012), «Planning, budgeting and disbursing funds for newborn survival in Katsina State, Nigeria - a Net-Map analysis ", Net-Map Toolbox, Washington, DC.

Schiffer, E. (2012), " Using Net-Map to assess and improve agricultural innovation systems ", in Agricultural Innovation Systems: An Investment Sourcebook, Banque mondiale, Washington, DC, pp. 593-597.

OCDE (2009), Régions et croissance : Une analyse des tendances, Éditions OCDE, Paris, http://dx.doi.org/10.1787/9789264056541-fr.

OCDE/CSAO (2017), Coopération transfrontalière et réseaux de gouvernance en Afrique de l'Ouest, Cahiers de l'Afrique de l'Ouest, Éditions OCDE, Paris, http://dx.doi.org/10.1787/9789264265974-fr.

USAID (2015), Report on road harassments, February, United States Agency for International Development, Washington DC.

Walther, O. (2015), «Business, brokers and borders: The structure of West African trade networks », The Journal of Development Studies, vol. 51, n 5, pp. 603-620.

Walther, O. (2014), "Trade networks in West Africa: A social network approach », The Journal of Modern African Studies, vol. 52, n², pp. 179-203. 



\section{DANS LA MÊME COLLECTION :}

Allen, T. et P. Heinrigs (2016), « Les nouvelles opportunités de l'économie alimentaire ouest-africaine »), Notes ouest-africaines, $\mathrm{N}^{\circ} 1$, Éditions OCDE, Paris. http://dx.doi.org/10.1787/5jlwjg67125f-fr

Lewis, K. et C. Buontempo (2016), « Climate Impacts in the Sahel and West Africa: The Role of Climate Science in Policy Making »), Notes ouest-africaines, N², Éditions OCDE, Paris. http://dx.doi.org/10.1787/5jlsmktwjcd0-en

Gnisci, D. (2016), « Women's Roles in the West African Food System: Implications and Prospects for Food Security and Resilience »), Notes ouest-africaines, N³3, Éditions OCDE, Paris. http://dx.doi.org/10.1787/5jlpl4mh1hxn-en

Staatz, J. et F. Hollinger (2016), « West African Food Systems and Changing Consumer Demands », Notes ouest-africaines, $N^{\circ} 4$, Éditions OCDE, Paris. http://dx.doi.org/10.1787/b165522b-en

Prieto Curiel, R., P. Heinrigs et I. Heo (2017), “ Cities and Spatial Interactions in West Africa: A Clustering Analysis of the Local Interactions of Urban Agglomerations m, Notes ouest-africaines, $N^{\circ} 5$, Éditions OCDE, Paris. http://dx.doi.org/10.1787/57b30601-en

En savoir plus : lia.beyeler@oecd.org 